\title{
Editorials
}

\section{Cannabis in primary care}

The reclassifying of cannabis to a schedule 2 drug in November 2018 was seen as a landmark moment for patients, indicating a shift in medical policy, and more importantly, attitude toward medicinal cannabis within the UK. Following a change in legislation and subsequent guidance from the Royal College of Physicians and the British Paediatric Neurology Association, the RCGP issued its own desktop guidance for GPs to enable informed discussions with patients around this subject matter.' Draft National Institute for Health and Care Excellence guidelines on cannabis-based medicinal products were released in August 2019, with full publication expected towards the end of this year.2

Despite an increasing number of patients enquiring about medical cannabis, the reality is that it remains a contentious subject, generally poorly understood by doctors and largely unavailable for patients. The reasons are complex and multifactorial and include there being a lack of physician education in prescribing; a lack of cannabis-based medicines available in the UK; a convoluted regulatory process in obtaining a medical cannabis prescription and suitable product; and the added fact that only GMC registered specialists may initiate a prescription.

Current UK legislation and practice guidelines which exclude GPs as primary first prescribers risks leaving GPs out of the conversation, despite the level playing field regarding the knowledge of cannabis medicines shared by both specialists and GPs alike. The authors propose that GPs are perhaps the best placed of all doctors to prescribe medical cannabis, as they do in most other countries where cannabis for medical purposes is now legal.

\section{CANNABIS AS A MEDICINE}

The cannabis plant contains a group of active plant chemicals called cannabinoids, including the most well-known THC (tetrahydrocannabinol) and CBD (cannabidiol), which have a modulatory effect on our endogenous endocannabinoid system, helping to regulate a range of major functions including our sleep/wake cycles, appetite, and mood, as well as numerous immune functions and anti-inflammatory effects. ${ }^{3}$ At higher doses, THC does have intoxicant properties, but it can also be useful medically at carefully monitored doses and combined with CBD to reduce psychoactive effects. It may be useful for many difficult-to-treat

\section{Cannabis for medical purposes is now legal in over 50 countries worldwide}

symptoms including chronic pain, spasticity, and nausea and vomiting, when other drugs have failed, side effects are intolerable, or an adjunct is needed to enhance efficacy. In the management of anxiety and epilepsy, CBD has been shown to be effective based on current preliminary research. ${ }^{4,5} \mathrm{~A}$ recent report by the World Health Organization showed CBD to exhibit no effects indicative of any abuse or dependence potential, as well as being generally well tolerated with a good safety profile. ${ }^{6}$

Many patients have become more aware of the therapeutic effects of cannabis and are accessing this illicitly through the black market. In addition to the risk of prosecution and exposure to criminality, patients using illicit street cannabis may unknowingly be consuming high levels of THC as well as a number of other impurities. A recent study found that the UK's cannabis street market is now populated by high-potency varieties of cannabis, which may have concerning implications for public health.?

\section{GPs AS PRESCRIBERS}

GPs could prescribe medicinal cannabis as they are a first point of contact for patients and are often the primary doctor involved with overseeing and coordinating medical treatments, as well as managing day-today symptoms. GPs tend to have a deep understanding of complex overlapping chronic illnesses and the quality of life issues facing patients who have exhausted all conventional drug options. They may also take on a more holistic approach to care rather than focusing on one system or disease in isolation.

Cannabis medicines using full spectrum herbal cannabis products, often used in oral oil form and referred to as 'full extract cannabis oils', require an individualised approach to dosing and monitoring, and it can take weeks or even months in some cases to find the optimal regime of cannabis medicine, and optimum ratios of $\mathrm{THC}$ versus $\mathrm{CBD}$. From a practical point of view, particularly within the NHS, GPs rather than specialists are perhaps better placed for regular follow ups to oversee close monitoring and make these subtle dose adjustments.

\section{CANNABIS AND PAIN}

One of the most commonly requested conditions for medical cannabis is for chronic pain, and a strong body of evidence is emerging for cannabis as an effective and alternative therapy for pain. 8.9 GPs are often the first point of contact for patients suffering with pain and commonly prescribe opiatebased medications. Although opioids for acute pain and palliative care can be effective, there is now strong evidence demonstrating that opioids are not a safe and effective treatment for most types of chronic pain, and may expose patients to significant harm. ${ }^{10}$ The reality is that an alarmingly high number of prescriptions continue to be issued, with the UK now having one of the world's fastest growing rates of opioid use. ${ }^{11}$ Gabapentinoids, which are also widely prescribed for pain, have recently been linked to new risks, including increased suicidal behaviour and unintentional overdoses. ${ }^{12}$

The difficulty for doctors is that chronic pain can be notoriously difficult to treat and can have devastating impact on both physical and mental health, and overall quality of life. Patients who are referred to specialist pain clinics by GPs may find themselves waiting for months to be seen while they continue to suffer with poorly controlled symptoms. Once they are seen, outcomes from specialist pain clinics are often variable. Unlike opioids, cannabis is safe in overdose with no reported deaths and carries a lower addiction risk. Cannabis is also known to have opioid sparing properties which may allow patients to safely reduce dosages of stronger opioid medication. ${ }^{13-15}$ A reduction of unnecessary prescriptions could, in turn, lead to the additional benefit of cost savings for the NHS.

\section{COMPASSIONATE CARE AND QUALITY OF LIFE}

Cannabis should be considered on a casespecific basis to help relieve unnecessary suffering and enhance overall quality of life where more conventional medications and therapies have failed. Many GPs will know a number of patients who are suffering with debilitating conditions, such as neurodegenerative disorders or cancer, 
who have not responded well to conventional treatments or are suffering the side effects of treatment, and it seems entirely appropriate that medical cannabis could be offered as an alternative or add-on for symptom control. Current poor patient access has meant a number of desperate patients and families are travelling abroad to receive medical cannabis prescriptions, often at great financial and personal cost. ${ }^{16}$

Over recent years there has been an emphasis on patient-centred care, where patients are involved in a decision-making process with their doctors, sharing goals and targets for treatments. This could be key to medical cannabis treatments where patients are informed of levels of evidence, scientific data, and efficacy, and play a more active role in the decision making process for an offlicence prescription.

\section{THE EVIDENCE BASE}

Studies of the cannabis plant have been restricted traditionally due to prohibition throughout most of the 20th century. Traditional randomised controlled studies may not be the most suitable for assessing whole plant cannabis-based medicines, as they contain not just one or few but hundreds of biologically-active compounds, and have multiple receptor targets in the brain and body, as well as other neurotransmitter systems and orphan receptors. It is likely that we may see a large body of evidence formed from longitudinal observational studies rather than from the sole reliance on randomised controlled trials.

There is also the consideration of the so-called 'entourage effect', where whole plant medicines are often more effective clinically than a single chemical isolated from the plant. The effects of medical cannabis may vary considerably between individuals, as well as the type of medical cannabis product used, and the dose and mode of administration. Medical cannabis treatments in many countries revolve primarily around using whole plant cannabis products for this very reason.

\section{EDUCATION}

Education programmes for medical practitioners must now be a top priority, and the authors would urge the UK's medical institutions to endorse high standard accredited teaching programmes. The teaching of the endocannabinoid system should also become a standard part of the medical school curriculum so that the next generation of doctors are competent in understanding this developing field of medicine.
Our priority should now be to look to work collaboratively within the UK and beyond to deepen our knowledge and application of this evolving field. The Medical Cannabis Clinicians Society (mww.ukmccs.org) has recently been set up with this in mind, as a non-profit society, with a focus on education, seminar events, and peer support for clinicians. It is open to all doctors, healthcare professionals, and medical students within the UK.

\section{CONCLUSION}

Cannabis for medical purposes is now legal in over 50 countries worldwide, and this number is increasing month by month. Those countries that have invested heavily in research, education, and infrastructure, are now establishing themselves as global leaders in this field, with positive clinical results for patients and economic benefits, with GPs playing a significant role in this development. The authors hope that with proper education and policy reform, the UK will be able to follow suit and offer this new class of medicines to improve the lives of patients who need it the most.

\section{ADDRESS FOR CORRESPONDENCE}

\section{Leon Barron}

\section{Email: leon.barronanhs.net}

\section{Leon Barron,}

Executive Committee Member, The Medical Cannabis Clinicians' Society; Locum \& Portfolio GP, London, UK

\section{Dani Gordon,}

Vice Chair, The Medical Cannabis Clinicians Society; GP, Integrative Medicine Physician, Educator, Writer, and Broadcaster, London, UK

\section{Provenance}

Freely submitted; externally peer reviewed.

\section{Competing interests}

Leon Barron has provided unpaid, voluntary medical advice to The Medical Cannabis Clinics and The Academy of Medical Cannabis (to June 2019). Since writing the editorial he has set up The Primary Care Cannabis Network, an education platform for GPs, with no industry links or commercial involvement. Dani Gordon has provided consultancy advice for companies working on medical uses of cannabis, and is the author of a forthcoming book. The CBD Bible.

DOI: https://doi.org/10.3399/bjgp19X706673

\section{REFERENCES}

1. Royal College of General Practitioners. Cannabisbased medicines: an interim desktop guide. 2018. https://uww.rcgp.org.uk/-/media/Files/CIRC/ Desktop-guides/Cannabis-based-medicationdesk-guide-nov-2018.ashx?la=en laccessed 4 Nov 2019).

2. The National Institute for Health and Care Excellence. Guideline: cannabis-based medicinal products. Draft for consultation, August 2019. 2019. https://uww.nice.org.uk/guidance/gidng10124/documents/draft-guideline laccessed 4 Nov 2019).

3. Russo EB, Marcu J. Cannabis pharmacology: the usual suspects and a few promising leads. $A d v$ Pharmacol 2017; 80: 67-134.

4. Blessing EM, Steenkamp MM, Manzanares J, Marmar CR. Cannabidiol as a potential treatment for anxiety disorders. Neurotherapeutics 2015 12(4): 825-836.

5. Lattanzi S, Trinka E, Russo E, et al. Cannabidiol as adjunctive treatment of seizures associated with Lennox-Gastaut syndrome and Dravet syndrome. Drugs Today (Barc) 2019; 55(3): 177-196.

6. World Health Organization. Cannabidiol (CBD) pre-review report. 2017. https://wnw.who.int/ medicines/access/controlled-substances/5.2 CBD.pdf (accessed 4 Nov 2019).

7. Potter DJ, Hammond K, Tuffnell S, et al. Potency of $\Delta^{2}$-tetrahydrocannabinol and other cannabinoids in cannabis in England in 2016 : implications for public health and pharmacology. Drug Test Anal 2018; 10(4): 628-635.

8. National Academies of Sciences, Engineering, and Medicine. The health effects of cannabis and cannabinoids: the current state of evidence and recommendations for research. Washington, DC: National Academies Press, 2017. DOI: https://doi. org/10.17226/24625

9. Davies S. Cannabis scheduling review part 1: the therapeutic and medicinal benefits of cannabis based products - a review of recent evidence. 2018. https://unw.gov.uk/government/ publications/cannabis-scheduling-review-part-1 (accessed 4 Nov 2019).

10. Faculty of Pain Medicine. A structured approach to prescribing. 2019. https://wnw.rcoa.ac.uk/facultyof-pain-medicine/opioids-aware/structuredapproach-to-prescribing laccessed 4 Nov 2019).

11. Organisation for Economic Co-operation and Development (OECD). Addressing problematic opioid use in OECD countries. 2019. http:// uww.oecd.org/health/addressing-problematicopioid-use-in-oecd-countries-a18286f0-en.htm laccessed 4 Nov 2019].

12. Molero Y, Larsson H, D'Onofrio BM, et al. Associations between gabapentinoids and suicidal behaviour, unintentional overdoses, injuries, road traffic incidents, and violent crime: population based cohort study in Sweden. BMJ 2019; 365: 12147.

13. Boehnke KF, Litinas E, Clauw DJ. Medical cannabis use is associated with decreased opiate medication use in a retrospective cross-sectional survey of patients with chronic pain. J Pain 2016; 17(6): 739-744.

14. Haroutounian S, Ratz Y, Ginosar Y, et al. The effect of medicinal cannabis on pain and quality-of-life outcomes in chronic pain: a prospective openlabel study. Clin J Pain 2016; 32(12): 1036-1043.

15. Nielsen S, Sabioni P, Trigo JM, et al. Opioidsparing effect of cannabinoids: a systematic review and meta-analysis. Neuropsychopharmacology 2017; 42(9): 1752-1765

16. Busby M. Mother of girl with epilepsy has supply of medical cannabis confiscated. The Guardian 2019; Apr 6: https://www.theguardian.com/society/2019/ apr/06/mother-of-epileptic-girl-teagan-emmaappleby-has-supply-of-medical-cannabisconfiscated (accessed 5 Nov 2019). 